\title{
Staatsbürgerschaft, Gender und globale Ungleichheiten
}

Der Paß ist der edelste Teil von einem Menschen. Er kommt auch nicht auf so einfache Weise zustand wie ein Mensch. Ein Mensch kann überall zustandkommen, auf die leichtsinnigste Art, aber ein Paß niemals. Dafür wird er auch anerkannt, wenn er gut ist, während ein Mensch noch so gut sein kann und doch nicht anerkannt wird.

- Bertolt Brecht: Flüchtlingsgespräche

Dass es so etwas gibt wie ein Recht, Rechte zu haben - und dies ist gleichbedeutend damit, in einem Beziehungssystem zu leben, in dem man aufgrund von Handlungen und Meinungen beurteilt wird -, wissen wir erst, seitdem Millionen Menschen aufgetaucht sind, die dieses Recht verloren haben und zufolge der neuen globalen Organisation der Welt nicht imstande sind, es wiederzugewinnen.

- Hannah Arendt: Elemente und Ursprünge totaler Herrschaft

\section{Einleitung}

In den gegenwärtigen Debatten um den Umgang mit Geflüchteten in Europa erscheinen Ungleichheiten von Staatsbürgerschaft und Geschlecht als zentrale Dimensionen, entlang derer vermeintliche Differenzen zwischen Aufnahmegesellschaften und Migrant_innengruppen verhandelt werden müssen. Im politischen Diskurs finden wir dieses Muster in der diskursiven Konstruktion von »sicheren Herkunftsländern«, von sogenannten Balkanflüchtlingen als Sozialschmarotzer und Kriminelle und von der "neuen Qualität sexueller Gewalt«, die von männlichen Geflüchteten ausgehe. Medial verhandelt wird diese Differenzierung unter anderem über eine deutsche / europäische Selbstinszenierung als zivilisatorisch und moralisch überlegen ob verwirklichter Frauen- und Homosexuellenrechte. Neuerdings schreiben selbst die CSU und Pegida diese als Maßstab für die Integration von Geflüchteten (weniger als eigenen Maßstab) ins Programm.

Dabei verweist der heutige Umgang mit Migration und Staatsbürgerschaft in der EU auf eine lange Tradition der Selbsterzählung Westeuropas als Ursprung von Modernität, Fortschritt und zivilisatorischer Überlegen- 
heit. Deren jetzige Anrufung hat ein Wiedererstarken kolonialer Genderund Citizenship-Arrangements zur Folge - verschiedene nationale/ ethnisierte Männlichkeiten und Frauenkörper werden von Seiten rechter Parteien gegeneinander ausgespielt, indem die "Silvesterereignisse von Köln« dazu instrumentalisiert werden, kolonial-rassistische Ängste vor "afrikanischen Vergewaltigern" zu schüren; Menschen- und Asylrechte zum "Schutz" von National- und EU-Grenzen werden eingeschränkt, während es geflüchteten Frauen und LGBT-Personen systematisch an geeigneten Unterkünften und Ausstattung fehlt - ihnen also der notwendige Schutz verwehrt oder der Familiennachzug für Geflüchtete ausgesetzt wird. Ein zunehmender Okzidentalismus findet sich im drastischen Erstarken rechtsnationaler Parteien in vielen Ländern und dem Ruf nach Grenzschließung und Abschiebung wieder. Die Sicherung abendländischzivilisatorischer Überlegenheit und Privilegien (bei Pegida bereits explizit im Namen verankert) - in der EU sowie in weiten Teilen der westlichen Welt - ist untrennbar verknüpft mit einem privilegiertem Staatsbürgerschaftsstatus und ökonomischem Wohlstand.

Wir gehen deshalb in diesem Beitrag der historischen Herausbildung von Ungleichheiten entlang der Dimensionen von Staatsbürgerschaft und Geschlecht nach, um zu zeigen, dass transregionale Verflechtungen von Ungleichheitsstrukturen seit der europäischen Kolonialexpansion durch okzidental-kapitalistische Vorstellungen von Geschlechterrollen, -normen und geschlechtlicher Arbeitsteilung begleitet wurden. Die Kategorie Gender hat die Institution der Staatsbürgerschaft seit ihrer Entstehung geprägt, und Gender und Staatsbürgerschaft waren auf hochkomplexe Weise verflochten mit anderen Dimensionen der Stratifizierung und Ungleichheit wie Rassisierung und Versklavung. Zugleich stellt das zugeschriebene Merkmal Staatsbürgerschaft, so unsere These, neben Geschlecht eine ebenso wichtige Dimension weltweiter Stratifizierung dar wie soziale Mobilität. Die Aufrechterhaltung vergeschlechtlichter Ungleichheiten sowohl innerhalb europäischer Gesellschaften als auch zwischen Männern kolonisierter und kolonisierender Regionen in der longue durée hat dazu geführt, dass Vergeschlechtlichung und Staatsbürgerschaft (wie auch Vergeschlechtlichung von Staatsbürgerschaft) bis heute die bedeutendsten Faktoren für extreme Ungleichheiten zwischen Individuen in reichen und armen Ländern darstellen. Um diese These zu belegen, stellen wir in einem ersten Schritt eine globale und kolonialitätskritische Perspektive auf Staatsbürgerschaft und Geschlecht vor. Anschließend gehen wir vergeschlechtlichten Strategien zur Erlangung von Staatsbürgerschaft 
wie Heirat, Mutterschaft, und Sex-Tourismus nach. Anhand solcher acts of citizenship werden ungleiche Staatsbürgerschaftsrechte unterlaufen, um vergeschlechtlichte, koloniale und rassisierte Strukturen aufzurufen, wieder einzuschreiben, aber auch anzueignen. Der Beitrag schließt mit einem Plädoyer für die stärkere Einbeziehung von fortbestehenden kolonialen, intersektional gedachten Geschlechterverhältnissen in Analysen globaler Machtbeziehungen.

\section{Staatsbürgerschaft in globaler Perspektive}

Analysen von (globalen) Ungleichheiten beziehen sich meist auf Unterschiede hinsichtlich des Einkommens-, Reichtums- oder Bildungsniveaus sowie von Status oder Beruf. Demgegenüber richtet der vorliegende Beitrag den kritischen Blick auf die bedeutende Rolle von Geschlecht und Staatsbürgerschaft als entscheidende Stratifizierungsfaktoren. Im Zuge kolonialer Zuschreibungen erfolgte seit der europäischen Kolonialexpansion die Projektion und Anwendung okzidental-kapitalistischer Vorstellungen von Geschlechterrollen, -normen und geschlechtlicher Arbeitsteilung. Diese wurden sowohl auf weiße Frauen in den Zentren und den Peripherien des globalen Systems, als auch auf "feminisierte«, als nicht-weiß Rassisierte sowie auf rassisierte Frauen, ethnische Gruppen und koloniale Gesellschaften projiziert.

Als Institution moderner westlicher Gesellschaften wird Staatsbürgerschaft üblicherweise als Mechanismus der Verringerung sozialer Ungleichheiten verstanden. Indem sie allen Staatsbürgern die gleichen Rechte und somit theoretisch die gleichen Möglichkeiten zuweist, wirkt sie sozioökonomischen Disparitäten entgegen. Während die Klassiker der Soziologie an diesem Verständnis festhalten, wird diese Sichtweise im Zuge globaler Perspektivierungen zunehmend hinterfragt. Einer der ersten Autor_innen, die sich die Frage gestellt haben, wie sich die Institution der Staatsbürgerschaft auf globale Ungleichheiten auswirkt, war Rogers Brubaker in seinem 1992 erschienenen Buch Citizenship and Nationhood in France and Germany. Brubaker macht darauf aufmerksam, dass alle Staaten heute den Zugang zu ihrer Staatsbürgerschaft begrenzen - entweder durch das Kriterium der Geburt auf dem Staatsgebiet im Falle des Territorialprinzips (jus soli) oder durch das der Herkunft von mindestens einem Staatsbürger im Falle des Abstammungsprinzips (jus sanguinis). Auf sehr willkürliche Weise, nämlich durch Zuschreibung bei Geburt (und von Geburt an), wird damit der Zugang von Staatsbürgern zu Gemeingütern, Arbeitsmärkten, 
Infrastruktur und sozialen Sicherungssystemen sehr unterschiedlichen Umfangs und Qualität reguliert. Als "Geburtsrechtslotterie«" bezeichnete deshalb die Rechtswissenschaftlerin Ayelet Shachar (2009) im Anschluss an Brubaker die heutige Logik der Gewährung von Rechten in einer politischen Gemeinschaft. Sie machte damit auf die bedeutende Rolle aufmerksam, die der Institution der Staatsbürgerschaft bei der Reproduktion globaler Ungleichheiten zukommt. Ihr zentrales Argument lautet, dass Staatsbürgerschaft ein sorgfältig reguliertes System ist, das den Zugang zu knappen Ressourcen auf die snatürlichen Erben der politischen Gemeinschaft beschränkt. Dies trifft sowohl für knappe Ressourcen wie Einkommen, die zwischen reichen und armen Ländern stark differieren, als auch und in dramatischerer Weise - für den Zugang zu lebensnotwendigen Ressourcen wie sauberem Wasser, medizinischer Versorgung und Nahrungsmitteln zu, bei dem der Zufall der Geburt in einem reichen Land erheblich mitentscheidet.

Shachars provokante These lautet, dass eines der zentralen Ungleichheitsmerkmale auf globaler Ebene - die Mitgliedschaft in der politischen Gemeinschaft eines Staates oder die Staatsbürgerschaft - analog zum mittelalterlichen Lehnswesen funktioniert. Sie wird ähnlich wie Erbeigentum (inherited property) von einer Generation zur nächsten - und unabhängig von Leistung - automatisch weitergegeben und stellt damit kein erworbenes, sondern ein eminent zugeschriebenes Merkmal dar. Als solches hat sie erhebliche Konsequenzen für die Lebenschancen derjenigen, die davon betroffen sind - sei es als Inkludierte oder als Exkludierte. Dabei seien jus sanguinis (das Abstammungsprinzip) und jus soli (das Territorialprinzip) für die Chancenverteilung innerhalb der globalen Ungleichheitsstruktur ähnlich folgenschwer.

Eine ähnliche Naturalisierung erfuhr Geschlecht als zugeschriebene und ungleiche Kategorie. In Bezug auf Migration und globale Ungleichheiten sind beide Kategorien eng und untrennbar - intersektional - miteinander verknüpft. Ungleiche und vergeschlechtlichte Organisationsformen korrespondierten mit einer strukturellen Verteilung von ungleichen Staatsbürgerschaftsrechten. Diese haben die moderne/koloniale Institution von Staatsbürgerschaft seit ihrer Einführung geformt und werden in neuen Formen und Vorstellungen von Staatsbürgerschaft neu aktiviert und artikuliert. Die Kontinuität zwischen den sukzessiven Mechanismen der Vergeschlechtlichung und ihren unterschiedlichen Ausprägungen in Westeuropa gegenüber den (ehemaligen) Kolonien beruht auf Staatsbürgerschaft

1 Alle Übersetzungen sind unsere eigenen. 
als zentrale Institution, welche Ungleichheiten zwischen Männern und Frauen, aber auch zwischen Moderne und Kolonialität ("globalem Norden" und "globalem Süden") aufrechterhält, eng verknüpft mit unterschiedlichen Formen der Rassisierung, Sexualisierung und Prekarisierung. Insbesondere das Konzept der Vergeschlechtlichung (gendering, nach Sheila Pelizzon 1998) umfasst in diesem Zusammenhang als Oberbegriff eine Vielzahl transregionaler Prozesse. Anhand gegenwärtiger Phänomene werden Artikulationen von Vergeschlechtlichung deutlich, die sich in Form von "sexuellem Exzeptionalismus" (Dietze 2015) äußern. Demzufolge imaginiert sich der "Westen« (oder »das Abendland«) als "zu Ende emanzipiert" (Dietze 2010) und projiziert geschlechteremanzipatorische Rückständigkeit, Sexismus, sexuelle Gewalt und Homophobie auf ein "nicht-westliches" (muslimisches) Anderes, in diesem Fall die durch diesen Diskurs als Bedrohung weißer westlicher Weiblichkeit und sexueller Freiheit konstruierten "nordafrikanischen Männern«. Wie stark die Stratifizierungsachsen Staatsbürgerschaft und Geschlecht/Vergeschlechtlichung miteinander verwoben sind und einander gar bedingen können, zeigt die sofort einsetzende Diskussion darum, die vermeintlichen Herkunftsländer dieser »bedrohlichen« Männlichkeit zu »sicheren Herkunftsländern« zu erklären und somit strukturell vom Grundrecht auf Asyl auszuschließen.

\section{Die Kolonialität von Staatsbürgerschaft und Geschlecht}

Eine globale Perspektive auf Staatsbürgerschaft zeigt, dass die Institutionalisierung von Staatsbürgerschaftsrechten in westlichen Nationalstaaten mit dem legalen (und physischen) Ausschluss nicht-europäischer, nicht-weißer und nicht-westlicher Bevölkerungen von zivilen, sozialen und kulturellen Rechten einherging. Die von zahlreichen Forscherinnen als koloniale Konstruktion entlarvte Kategorie Gender (McClintock 1995, Stoler 1997, Lugones 2007) hat die moderne/koloniale Institution der Staatsbürgerschaft seit ihrer Entstehung geprägt. Frauen wurden Staatsbürgerschaftsrechte sowohl innerhalb als auch außerhalb des Westens nur schrittweise erteilt, während Männer und Frauen anderer Regionen entlang kolonialer Linien vergeschlechtlicht worden sind. Ansätze zur Kolonialität (Quijano 2000, Mignolo 2000, Lugones 2007 und 2008) thematisieren, zunächst für den Kontext Lateinamerikas, die strukturelle Verteilung ungleicher, vergeschlechtlichter Staatsbürgerschaftsrechte als bedeutsame Komponente von Modernität / Kolonialität, für den wir den Begriff der Kolonialität von Staatsbürgerschaft verwenden (vgl. Boatcă / Roth 2016). 
Zeitgleich mit dem Aufkommen der westeuropäischen Institution der Staatsbürgerschaft - als Ausdruck von Freiheit und dabei als Gegenteil von Versklavung - wurden formell die Rechte von Frauen männlicher Autorität unterworfen, und ihre Mobilitätsrechte entsprechend eingeschränkt. Im direkten Anschluss an die Französische Revolution wurden Staatsbürgerschaftsrechte lediglich männlichen Eigentümern zugesprochen, deren Fähigkeit, Steuern zu zahlen und Militärdienst zu leisten und somit zur Aufrechterhaltung der sozialen Ordnung beizutragen, sie als »aktive Bürger« qualifizierte. Frauen, Ausländer und Kinder wurden hingegen als "passive Bürger definiert, und von allen politischen Rechten ausgeschlossen (Blackburn 1988, 198; Wallerstein 2003, 653f.). Während dies für weiße westeuropäische Frauen als temporäre Maßnahme galt, die durch weitere Bildungsmaßnahmen überwunden werden sollte, sprach die Reduktion versklavter Afrikaner/innen zu Waren und "pure flesh " (Broeck 2006 , n.p.) diesen auch jeden Gender-Status ab, der mit dem von Staatsbürgern vergleichbar wäre. ${ }^{2}$

Sowohl Olympe de Gouges in ihrer "Declaration of the Rights of Women and the Female Citizen" (1791) als auch Mary Wollstonecrafts »A Vindication on the Rights of Women" (1792) verwiesen auf die männliche Dividende des Menschenrechts- und des Staatsbürgerschaftsdiskurses ohne absehbaren Erfolg. Die französische Verfassung von 1793 weitete aktive Staatsbürgerschaft auf alle erwachsenen (nicht notwendig besitzenden) Männer aus. Damit wurde die Mitgliedschaft von Frauen in der sozialen Gemeinschaft von ihrer Beziehung zu Männern abhängig gemacht und sie wurden zunehmend aus der männlichen Domäne der Kultur ausgeschlossen (Hanagan 2002, 167). ${ }^{3}$ Ihre im bürgerlichen Milieu im westeuropäischen Kontext kulturell und normativ verbindliche Zuweisung an die private Sphäre des Haushalts ging mit der gleichzeitigen Konstruktion der

$2 »[\ldots]$ enslaved African-origin female beings never qualified as women (because of their non-humanness, it followed logically) in the Euro-American modern world, and therefore were not interpellated to partake in the ongoing social construction and contestation of gender [...] - a category that would have enabled a black female claim on social negotiations did not apply to things, to what was constructed as and treated as human flesh. Moreover, that very category gender emerged in western transatlantic rhetoric precisely in the context of creating a space for white women, who refused to be treated like slaves, like things." (Broeck 2008, 15)

3 Zahlreiche afroamerikanische und afrokaribische, zumeist ehemals versklavte Frauen, wiesen schon früh auf die gleichzeitige Artikulation von Gender und »Race» sowie ihre vollkommen unterschiedliche vergeschlechtlichte Positionierung entlang einer kolonialen/rassistischen Bresche und klagten eine intersektionale Perspektive ein. Das bekannteste Beispiel ist Sojourner Truths Intervention bei der Women's Rights Convention in Akron, Ohio 1953 mit ihrer Rede "Aren't I a Woman?». 
dort verübten unbezahlten Arbeit als natürliche Ressource und deshalb als "Nicht-Arbeit« einher.

Pnina Werbner und Nira Yuval-Davis beschreiben den Ausschluss von Frauen von Staatsbürgerschaftsrechten entsprechend als »intrinsisches Merkmal ihrer Naturalisierung als Verkörperung des Privaten, des Familiären und des Emotionalen" und damit als «grundlegend für die Konstruktion des öffentlichen Raumes als maskulin, rational, verantwortungsvoll und respektabel" $(1999,6)$. Indem Frauen, Kinder und Ausländer so auf die (wenn auch jüngste) Vergangenheit des Zivilisierungsprozesses zurückgestuft wurden, den Männer vermeintlich durchlaufen hatten, schaffte die Implementierung von vorgeblich universellen Prinzipien konstant rassisierte und vergeschlechtlichte Partikularismen. Die entsprechenden Ausschlüsse reichten "von kolonialen Subjekten zu Frauen, bestimmten Klassen und rassisierten Minderheiten bis hin zu Menschen mit anderen Sexualitäten und Fähigkeiten« (Dobrowolsky / Tastsoglou 2006, 10).

\section{Genealogien kolonialrassistischer, vergeschlechtlicher Arbeitsteilung}

Wie erwähnt, hat die Kategorie Gender die moderne/koloniale Institution der Staatsbürgerschaft seit ihrer Entstehung geprägt. In ihrem Essay "The Traffic in Women" (1975) beschreibt die feministische Forscherin Gayle Rubin das "Sex-Gender-System" als eine "Anordnung von Arrangements" (set of arrangements) durch die Sex in Gender übersetzt wird und welches als Prototyp für alle sozialen und ökonomischen Beziehungen dient. ${ }^{4}$ Im Rahmen dieses Systems tauschen Männer Frauen untereinander aus entlang eines Kontinuums, das von Prostitution bis zu Heirat reicht (vgl. Rubin 1975). Rubin betrachtete das Sex / Gender-System als der kapitalistischen Ökonomie zugehörig, das aber durch die gesamte Menschheitsgeschichte hindurch das Gewebe der Kultur bildete. Jean Franco argumentiert demgegenüber, dass bestimmte Formen des "Austauschs« kolonisierter Frauen - etwa versklavte indigene Frauen, die den spanischen Eroberern als Geschenk vermacht oder zwischen aztekischen und spanischen Männern ausgetauscht wurden - bereits integraler Bestandteil der

4 In den Amerikas kämpften schon frühe Feministinnen gegen die automatische Bindung der Frauen an den Ehemann mittels Staatsbürgerschaft (Frauen verloren ihre Staatsbürgerschaft, wenn sie einen Mann mit anderer Nationalität heirateten). Vgl. z.B. die Interventionen von Feministinnen bei den Inter-American Conferences (Forestell / Moynagh 2012). 
Eroberung waren (1999, 71 f.). ${ }^{5}$ Indem sie eine Weltsystem- und eine dekoloniale Analyse (Wallerstein 1974, Mignolo 2000) mit einer feministischen Perspektive verknüpfte, prägte María Lugones den Begriff des »modernen / kolonialen Gender-Systems" (modern/ colonial gender system), um auf die wechselseitige Konstituierung von Gender, Modernität und Kolonialität im Kapitalismus zu verweisen:

Colonialism did not impose precolonial, European gender arrangements on the colonized. It imposed a new gender system that created very different arrangements for colonized males and females than for white bourgeois colonizers. Thus, it introduced many genders and gender itself as a colonial concept and mode of organization of relations of production, property relations, of cosmologies and ways of knowing. (Lugones 2007, 186)

Die spezifische Verknüpfung von Rassisierung und Vergeschlechtlichung wurde im Kontext des Sklavereisystems in den europäischen Kolonien besonders deutlich. Indigene und Versklavte blieben von den Staatsbürgerschaftsrechten der Kolonisierer ausgeschlossen, Regelungen und Gesetzgebungspraktiken erfolgten willkürlich. So lange der Import Versklavter kostengünstiger war als die natürliche Reproduktion von Arbeitskraft, hatte man versklavte Frauen in der Karibik systematisch davon abgehalten, Familien zu gründen und Kinder zu bekommen (Mies 1996, 91 ff.). Nach der Abschaffung des transatlantischen Sklavenhandels 1807 wurde hingegen das local breeding zu einer lukrativeren Option. In Übereinstimmung mit der Zivilisierungsmission der Kolonisierer war die Einführung der bürgerlichen Ehenorm die adäquate Politik gegen die vermeintliche Primitivität sindigener Geschlechterbeziehungen. Der Widerstand der ehemals Versklavten gegen die plötzliche Aufoktroyierung der monogamen Norm der Kolonisierenden, der Kernfamilie oder von beidem, ging mit hohen ökonomischen Kosten für die Kolonialverwaltung einher. ${ }^{6}$ Ins-

5 Franco beschreibt "gehandelte«/ «ausgetauschte« Frauen wie die Malinche in Mexiko als Repräsentationen der "Verkörperung der Eroberung" - das Andere als feminisiert - und behauptet, dass derartige koloniale Praktiken des Frauenhandels "sowohl die notwendigen sexuellen als auch praktischen Dienste" für die Bevölkerung der Neuen Welt und die Gründung einer mestizo Bevölkerung bereitstellten (Franco 1999: 76). Franco betrachtet diese Praktiken als den "Anfang vom Ende der Geschenkökonomie« und als Übergang zum vertraglich geregelten Handel/ Austausch (Franco 1999: 74).

6 Praktiken und Gesetze bezüglich der Heirat von versklavten Personen variierten stark, in den USA wurde Versklavten z.B. die gesetzliche Ehe verwehrt (vgl. Goring 2006). Da sie als Eigentum ihrer Besitzer galten, und vom sozialen und sogar menschlichen Status (einschließlich dem Gender-Status) der Sklavereigesellschaften ausgeschlossen blieben, waren versklavte Personen häufig willkürlichen Regeln und 
besondere aktive Geburtsstreiks versklavter Frauen - wie sie in der britischen Karibik zu Beginn des 19. und in Deutsch-Südwestafrika um die Wende zum 20. Jahrhundert aufkamen - hatten ernsthafte Auswirkungen auf die Pläne der Plantagenbesitzer, billige Arbeitskraft durch natürliche Reproduktion der ehemals Versklavten zu bekommen. Zugleich sorgten die staatlichen Gesetzgebungsmaßnahmen gegen sogenannte "gemischtrassige " Ehen, welche im Zuschnitt innerhalb der kolonialen Welt stark variierten, ${ }^{7}$ dafür, dass in den deutschen Kolonien in Afrika 1908 beide Partner und ihre Nachkommen ihre Staatsbürgerschaft und das Wahlrecht verloren. Gleichzeitig wurden informelle sexuelle Beziehungen zwischen Weißen männlichen Kolonisierern und Schwarzen Afrikanerinnen geduldet, solange aus diesen kein Nachwuchs hervorging (Mamozai 1982, 129). Beide Maßnahmen zielten darauf, die nicht-Weiße Bevölkerung vom Erwerb von Besitz abzuhalten, und ihnen damit auch das Wahlrecht vorzuenthalten. Der stärkste Nebeneffekt war aber, dass schwarze Frauen in Konkubinat und Prostitution gezwungen wurden, während sich weiße Frauen zunehmend auf Haushalt und Mutterschaft reduziert sahen (Bush 1981, Alexander 1991).

In gleichem Maße wie die Subsistenzproduktion außerhalb Europas zu »rückständiger« und »unterentwickelter« Arbeit erklärt wurde, ermöglichte das in Westeuropa propagierte bürgerliche Familienmodell, dass weibliche Hausarbeit als "nicht-Arbeit« stigmatisiert und Frauen insgesamt zur Norm nicht entlohnter Arbeit erklärt wurden.

Die aktuelle weltweit steigende Integration von Frauen in den Arbeitsmarkt bei gleichzeitiger Flexibilisierung und Prekarisierung von Arbeit verdeutlicht die Auswirkungen der veränderten Geschlechterrollen auf globale Dynamiken. Strategien zur Erlangung von Staatsbürgerschaft wie Heirat, Mutterschaft, und Sex-Tourismus dienen als Praktiken der Subversion ungleicher Staatsbürgerschaftsrechte. Auch im Rahmen dieser "acts of citizenship" (Isin / Nielsen 2008) werden vergeschlechtlichte, koloniale und rassisierte Strukturen häufig aufgerufen, wieder eingeschrieben, aber auch angeeignet und unterwandert.

Praktiken ausgesetzt (vgl. Andrews 2000 für verschiedene Praktiken und Rechtsnormen).

7 Morrison 2010 vergleicht Tabus und Gesetzgebung gegen "gemischtrassige« Ehen in Kuba, Brasilien und den USA im 19. Jahrhunderts. 


\section{Acts of Citizenship - "Global Care, Tourism, Marital Chains"}

Wie gezeigt, stellt neben Geschlecht das zugeschriebene Merkmal Staatsbürgerschaft eine ebenso wichtige Dimension weltweiter Stratifizierung dar wie soziale Mobilität. Die Zugehörigkeit zu gehobenen Klassen geht auf globaler Ebene mit dem bedeutsamen Vorteil einher, sowohl die $\mathrm{Zu}-$ schreibung als auch die eigentliche Migration zu umgehen. Ob die soziale Mobilität durch Erwerb des legalen Status in einem wohlhabenden Land als Migration zählt oder diese konstituiert/begründet, ist nicht nur abhängig vom Staatsbürgerschaftsstatus einer Person, sondern in erheblichem Maße auch von deren finanziellen Ressourcen. Deshalb stellt der Zugriff auf Marktmechanismen, um die Zuschreibung von Staatsbürgerschaft zu umgehen, eine immer stärker sichtbare, aber dennoch seltene Option dar, die nur wenigen Reichen offen steht. Ihre Mobilität wird jedoch selten als Migration bezeichnet - sie gelten als "globale Investoren", "Expats", oder "ausländische Ansässige aus Steuergründen« (foreign residents for tax purposes), während ihr Migrationsprozess häufig als relocation gilt oder als "Businessmigration" qualifiziert wird. Die in den letzten Jahren zu beobachtende Kommodifizierung von Staatsbürgerschaftsrechten weltweit, d.h. die Möglichkeit, Aufenthaltserlaubnisse und Staatsbürgerschaft käuflich zu erwerben, macht die Analogie von Staatsbürgerschaft und Eigentum besonders deutlich (vgl. Boatcă 2015, 189 ff.).

In Übereinstimmung mit der weltweiten Arbeitsteilung sind diejenigen wohlhabenden Individuen in hohen Positionen, die sich eine privilegierte Staatsbürgerschaft »erkaufen« können, hauptsächlich männlich. Die kolonial geprägte Vererbung von Eigentum unterstützt dabei das koloniale Erbe von privilegierter Staatsbürgerschaft westlicher Staaten entlang der weltweiten Gender-Dividende von Staatsbürgerschaft. Durch die weltweit steigende Integration von Frauen in den Arbeitsmarkt bei gleichzeitiger Flexibilisierung und Prekarisierung von Arbeit entstanden Phänomene, die aktuell unter Stichworten wie "Feminisierung der Arbeit», "Feminisierung der Armut" und "Feminisierung der Migration" diskutiert werden. ${ }^{8}$ Damit steht die Feminisierung der Armut im Zusammenhang mit transnationalen und globalen Prozessen wie der Feminisierung grenz-

8 Ursprünglich im Kontext von Industrienationen wie den USA und Deutschland thematisiert (vgl. Pearce 1978), werden die geschlechtsspezifischen Armutsrisiken von Frauen vermehrt im Hinblick auf ihre Diskriminierung auf dem Arbeitsmarkt, ihren eingeschränkten Zugang zu Ressourcen, Wissen oder Krediten in den 1990er Jahren auf ihre globale Bedeutung hin untersucht und durch Dokumente der Vereinten Nationen bekannt gemacht (Vereinte Nationen 2000). 
überschreitender Migration und der Feminisierung von Arbeit in prekären und nicht formalisierten Beschäftigungsverhältnissen, wie z.B. dem Bereich der privaten Haushalte. ${ }^{9}$ Im Zusammenhang mit der Feminisierung der Arbeit und der Migration wird neuerdings von einer "transnationalen Geschlechterordnung" gesprochen, die auf ethnisierten und rassifizierten Konstruktionen von Geschlecht beruht: So werden Frauen als besonders geeignet für mechanische Arbeiten am Fließband oder für Näharbeiten definiert - Tätigkeiten, die weltweit als niedrig qualifiziert erachtet und geringer entlohnt werden. Geschlecht erscheint aus dieser Perspektive als eine globale Strukturkategorie, die mit rassifizierten und ethnisierten Kategorien verknüpft und über lokale wie globale Arbeitsmärkte, Organisationen und Machtstrukturen vermittelt wird (vgl. Dannecker 2005, 280). Feminisierung ist also ein Indikator für eine Reihe von Ungleichheitsprozessen, die mit den Auswirkungen der ökonomischen Globalisierung für Frauen einhergehen: Während die Feminisierung der Armut die geschlechtsspezifischen Armutsrisiken von Frauen im Hinblick auf ihre Diskriminierung auf dem Arbeitsmarkt, ihren eingeschränkten Zugang zu Ressourcen, Wissen oder Krediten umfasst, bedingt sie im transnationalen und globalen Zusammenhang die Feminisierung von grenzüberschreitender Migration mit sowie die Feminisierung von Arbeit in prekären und nicht formalisierten Beschäftigungsverhältnissen. Die Feminisierung von Armut, Migration und Arbeit hat gravierende Auswirkungen auf die Flexibilisierung von Arbeitsverhältnissen und Staatsbürgerschaftspraktiken und auf die Ethnisierung und Rassisierung von Migrant/innen. Denn global gesehen führen Transformationen von Geschlechterordnungen zur Neuverteilung von Arbeit - hauptsächlich zwischen Frauen, jedoch auf einer globalen West-Ost bzw. Nord-Süd- Achse. In Form von »Global Care Chains « befriedigen Migrantinnen aus ärmeren Regionen die wachsende Nachfrage des globalen Nordens an Haus- und Pflegearbeit und übertragen ihre Pflegearbeit anderen Frauen vor Ort.

Der Titel des Buches von Barbara Ehrenreich und Arlie Russel Hochschild Global Woman. Nannies, Maids, and Sex Workers in the New Economy (2004) fasst die Hauptmöglichkeiten, welche die zunehmende Feminisierung von Migration (als nicht-weiß/nicht-westlich) rassisierten Frauen eröffnet, prägnant zusammen. Vergeschlechtlichte koloniale / globale Ungleichheiten spiegeln sich strukturell auch in globalisierten Heiratsmärkten

9 Neuere Arbeiten zeigen, dass die Feminisierung von Haushaltsarbeit (und darüber hinaus) nicht nur eine Frage der Quantität, d.h., des hohen Frauenanteils in diesem Bereich ist, sondern vielmehr eine Frage der Qualität, die dieser Arbeit zugeschrieben wird (vgl. Gutiérrez Rodríguez 2010: 101 f.). 
(vgl. 2014) und Sextourismus-Szenarien (vgl. O’Connell Davidson/Sánchez Taylor 1999) wider - stets auf der Folie der oben dargelegten Ungleichheiten bezüglich sozialer und physischer Mobilität, welche sich in gegenwärtigen spezifisch vergeschlechtlichten Staatsbürgerschaftspolitiken - bzw. Migrationspolitiken - ausdrückt.

\section{(Sex-)Tourismus, Heirat und Mutterschaft als "(Fast) Tracks" zu Staatsbïrgerschaft}

Anders als die vornehmlich männlichen Investoren, die Mobilität im Tausch für einen Scheck erhalten können, ${ }^{10}$ bieten arme Frauen, Transgender Personen und rassisierte Andere sich selbst zum Tausch an. Das heißt, sie bieten ihre Körper, ihre emotionale Arbeit, Hausarbeit, oder alles auf einmal, um sozialen Aufstieg durch Staatsbürgerschaft zu erreichen. An vielen Orten des globalen Südens stellt Tourismus eine der wenigen Formen des Zugangs zu harter Währung, Konsumgütern oder Luxusprodukten dar. In einigen Fällen eröffnen Begegnungen mit Tourist/innen sogar Aussicht auf Mobilität, wie eine Auslandsreise mittels eines Tourismusvisums, eine Aufenthaltserlaubnis, oder sogar eine Heirat und ein westlicher Pass. Nord-Süd Tourismus - und Sex- oder Romance-Tourismus ${ }^{11}$ im Besonderen - basiert jedoch auf zutiefst ungleichen Machtstrukturen. Wer wo Tourist oder Touristin sein kann, und von wem er oder sie bedient wird, ist mit sehr asymmetrischen und kolonialen Stratifizierungsachsen verknüpft, die stark von einer rassisierten und vergeschlechtlichten Dimension geprägt sind. Besitzer/innen eines westlichen Passes

10 Während sowohl Investorenstaatsbürgerschaft als auch Arbeitsmigration darauf ausgerichtet sind, dass mehr Menschen Zugang zu privilegierter Staatsbürgerschaft erhalten, zielt die rassisierte Kriminalisierung von Migrant/innen in den Kernregionen - vor allem in der EU und in den USA - nur auf letztere: Für Arbeitsmigrant/innen sind ererbte Staatsbürgerschaft und langwierige Einbürgerungsprozeduren rechtlich (wieder) als einzige Optionen durchgesetzt.

11 Als "Romance Tourism« bezeichnen einige Forscher/innen Tourismus von (vornehmlich heterosexuellen) Frauen in ärmere Regionen, der romantische und/oder sexuelle Beziehungen zu Einheimischen einschließt oder zum Ziel hat und mit dem Austausch von Geld, Konsumgütern oder Reiserleichterungen einhergeht. Der umstrittene - Begriff wird vom Begriff des »Sextourismus abgegrenzt, da zum einen die Touristinnen ihre Begegnungen im Narrativ von Urlaubsromanzen erzählen und dezidiert von (»männlichem») Sextourismus abgrenzen und zum anderen meist mehr als reine sexuelle Dienstleistungen mit festem Preis beinhaltet (etwa gemeinsames Ausgehen, Essen, Tanzen bis hin zur Begleitung während des gesamten Urlaubs) (vgl. Pruit/LaFont 1995; O'Connell Davidson 2001). Ein anschauliches Beispiel bietet Ulrich Seidls semidokumentarischer Film »Paradies: Liebe« (2013). 
(mit einem mittleren oder hohen Einkommen) können ihr Staatsbürgerschaftsprivileg nutzen, um z.B. in die Karibik zu reisen. In den meisten ehemals kolonisierten Regionen, die wirtschaftlich in starkem Maße von der Tourismusindustrie abhängig sind, sind Romance- und Sextourismus untrennbarer Teil des Traumurlaubs westlicher Reisender aller Geschlechter und sexueller Orientierungen (vgl. O’Connell Davidson/Sánchez Taylor 1999). ${ }^{12}$ Vor dem Hintergrund einer langen Tradition der Exotisierung und Sexualisierung des kolonialen "Anderen" (vgl. McClintock 1995) werden in diesen Szenarien unvorteilhafte Positionen hinsichtlich des Alters, Geschlechts und/oder der Klasse westlicher Männer und Frauen durchkreuzt vom "cultural / racial capital of whiteness" (Shohat/Stam 2012, 191), das ihnen als (Sex-) Tourist_in außerhalb des Westens qua Staatsbürgerschaftsprivileg zukommt. Sexarbeiter_innen an Urlaubsorten des globalen Südens können im Gegenzug ihr Staatsbürgerschaftsdefizit in erotisches Kapital umwandeln, das auf der kolonialen und rassisierten erotischen westlichen Imagination des schwarzen Körpers beruht und dadurch finanzielle Vorteile im Rahmen einer strukturell ungleichen Begegnung erzielen (vgl. Roth 2013). Die nicht-westlichen Partner_innen sind dabei jedoch in hohem Maße abhängig von der Gunst ihrer Partner_innen mit privilegiertem Pass. Frauen und andere Marginalisierte sind hier, einmal mehr, besonders häufig Opfer von psychischer oder sexueller Gewalt. ${ }^{13}$

Binationale Paare sind in den meisten westlichen Ländern über Jahre nach der Eheschließung ständig Misstrauen, Kontrollen und Illegalisierung ausgesetzt (Messinger 2013, 377). Strategien oder acts of citizenship zur sozialen Mobilität durch Heirat sind zum Ziel staatlicher Sanktionen geworden. Sie werden als eine Art "gefälschte Abstammung" (forged descent) (aus einer auf Blutsrecht basierenden Bürgergemeinschaft) in jus sanguinis Arrangements gebrandmarkt (Boatcă 2014). In zahlreichen europäischen Staaten - darunter Deutschland, Griechenland, Dänemark, Estland, Litauen, und Lettland - ist die rechtliche Aufenthaltsgenehmigung beider Partner die Voraussetzung für gleichgestellte (eingetragene) Partnerschaf-

Zu Sex Tourismusforschung und Sextourismus und Ungleichheiten, vgl. Sánchez Taylor 2010.

13 Darüber hinaus existieren Heiratsagenturen, die das "rassisch-erotische "Kapital von (zumeist) Frauen aus armen Ländern im Austausch gegen das "StaatsbürgerschaftsKapital« von Männern (und Frauen) des globalen Nordens anbieten, die Schwierigkeiten haben, in ihren Gesellschaften ein / en Partner / in zu finden oder Partner / innen mit speziellen Charakteristika suchen (z. B. eine deutlich jüngere, hingebungsvolle, fürsorgliche, attraktive Partnerin, die aus dem Katalog der Agentur ausgewählt werden kann). 
ten falls eine Partner_in EU-Bürger_in ist und der oder die andere nicht. In Deutschland und Österreich bezeichnet man weitläufig Ehen zwischen EU- und nicht-EU-Bürgerinnen und -Bürgern, die auf eine Aufenthaltsgenehmigung abzielen, abwertend als "Scheinehe«. Die Tatsache, dass der Begriff niemals auf Eheschließungen zwischen zwei westlichen Staatsbürger_innen - die auf Steuerersparnis oder auf andere staatliche Privilegien für verheiratete Paare abzielen könnten - angewendet wird, offenbart eine hohes $\mathrm{Maß}$ an institutionalisiertem Rassismus (Messinger 2013, 386). ${ }^{14}$ Verdächtige Ehepaare sind demütigenden staatlichen Kontrollen unterworfen - z. B. in Form unangekündigter Besuche zum Beweis der »Echtheit» der Beziehung. Irene Messinger zieht in ihrer Analyse (2013) des österreichischen Falls eine klare Verbindungslinie zwischen der Verschärfung von Migrationskontrollen in vielen EU-Staaten und der rechtlichen Situation binationaler Paare, bei denen einer der Partner kein EU-Staatsbürger ist. Die zutiefst vergeschlechtlichte, rassisierte und klassistische Dimension solcher staatlicher Überwachung binationaler Ehen ist offensichtlich: Österreichische Frauen mit niedrigem Einkommen, die mit afrikanischen Männern verheiratet sind, die das Land nicht legal betreten haben, werden am häufigsten verdächtigt. Die gleich große Gruppe österreichischer Männer, die mit asiatischen Frauen verheiratet sind, gilt hingegen nicht als verdächtig und wird kaum der Überwachung ausgesetzt. Darüber hinaus gilt der Altersunterschied nur dann als verdächtig, wenn die österreichische Frau erheblich älter ist. Außerdem werden die Chancen für untere Einkommensgruppen verringert, da Paare seit 2006 ein regelmäßiges Einkommen von über 1200 Euro vorweisen müssen.

Die beschriebenen Praktiken zeigen, wie Staatsbürgerschaftsregimes einerseits mittels intersektional vergeschlechtlichter Praktiken wie Tourismus und Heirat unterwandert werden. Andererseits werden anhand der Bewertungen und Sanktionierungen dieser Praktiken die engen Verflechtungen von Staatsbürgerschaft als zentrale Achse globaler Ungleichheiten und Vergeschlechtlichung deutlich.

14 Interessanterweise werden Eheschließungen zwischen deutschen (oder anderen) Staatsbürgern, und verfolgten Jüdinnen und Juden (denen während der Nazi-Diktatur die Staatsbürgerschaft entzogen wurde) heute als "Schutzehe« bezeichnet. (vgl. Messinger: 2012) 


\section{Ausblick}

Staatsbürgerschaft, die auf kolonialrassistischen und -ethnischen Konstrukten basiert, und Geschlecht/Gender, eine Kategorie, die im Zuge des Kolonialismus neu konfiguriert worden ist, sind bis heute Schlüsselkomponenten für das Fortbestehen globaler Ungleichheiten. Wie die erwähnten Beispiele verdeutlichen, treffen Versuche, die askriptive Logik von Staatsbürgerschaft mit ihren eigenen Waffen zu schlagen - zum Beispiel durch den Erwerb einer Staatsbürgerschaft durch Heirat - auf Kritik, auf Sanktionen und Kriminalisierung von Seiten supra-staatlicher und finanzieller Institutionen. Man setzt solchen Versuchen rechtliche Gegenmaßnahmen entgegen, überwacht und kontrolliert sie entlang rassisierter, ethnisierter und vergeschlechtlichter Linien. Da wohlhabende Mittelklassefamilien im globalen Norden von Migrant_innen zur Bewältigung von Kinderbetreuung, Hausarbeit, Altenpflege und sexuellen Dienstleistungen abhängig geworden sind, entsteht laut Ehrenreich und Hochschild, "eine globale Beziehung ..., die die traditionelle Beziehung zwischen den Geschlechtern widerspiegelt«:

The first world takes on a role like that of the old-fashioned male in the family-pampered, entitled, unable to cook, clean, or find his socks. Poor countries take on a role like that of a traditional woman within the family - patient, nurturing, and self-denying. A division of labor feminists critiqued when it was slocak has now, metaphorically speaking, gone global. (Ehrenreich und Hochschild 2004, 11 f.).

Die erwähnten Beispiele verweisen somit auf die (strukturell) koloniale Dimension und auf die intersektionale Verknüpfung von Geschlecht und Staatsbürgerschaft. Beide Kategorien sind historisch geworden, sie variieren von Kontext zu Kontext und sind von fortbestehenden kolonialen und intersektional vergeschlechtlichten, kapitalistischen Machtstrukturen geprägt. Zugleich bringen diese Strukturen auch alternative/subversive acts of citizenship hervor, um Zuschreibungen zu umgehen, wenngleich dies auf der Grundlage einer höchst ungleichen »Matrix" geschieht. Im Zuge der gegenwärtigen Debatten um den Umgang mit Geflüchteten in Europa werden sich die beschriebenen Staatsbürgerschafts- und GenderUngleichheiten - ebenso wie die Widerstandspraktiken dagegen - absehbar verstärken. Schon jetzt führen sie zu einem Wiedererstarken der hier besprochenen kolonialen Gender- und Citizenship-Arrangements. Vor diesem Hintergrund ist die Reflexion von fortbestehenden kolonialen Strukturierungsachsen in globaler Perspektive für die Analyse verge- 
schlechtlichter Machtbeziehungen dringend geboten. Dazu gilt es, Intersektionalität »translokal« weiterzudenken. Eine globale, intersektionale Perspektivierung von globalen Ungleichheiten und Migration ist notwendig, will man diesen besorgniserregenden Tendenzen entgegen wirken.

\section{Literatur}

Alexander, Jacqui M. (1991): Redrafting Morality: The Postcolonial State and the Sexual Offences Bill of Trinidad and Tobago. In: Chandra T. Mohanty/Ann Russo/Lourdes Torres. (Hrsg.): Third World Women and the Politics of Feminism. Bloomington, $133-152$.

Andrews, George Reid (2000): Afro-Latin America. Oxford.

Blackburn, Robin (1988): The Overthrow of Colonial Slavery, London: Verso.

Brubaker, Rogers (1992): Citizenship and Nationhood in France and Germany. Cambridge, MA.

Boatcă, Manuela (2015): Global Inequalities Beyond Occidentalism. Farnham.

Boatcă, Manuela (2014): Commodification of citizenship: Global inequalities and the modern transmission of property. In: Wallerstein, Immanuel/Chase-Dunn, Christopher/Suter, Christian (Hrsg.): Overcoming Global Inequalities. Boulder, CO, 3-18.

Boatcă, Manuela / Roth, Julia (2016): Unequal and Gendered: Notes on the Coloniality of Citizenship. In: Current Sociology, März 2016, 191-212.

Broeck, Sabine: „Enslavement as regime of western modernity: Re-reading gender studies epistemology through black feminist critique.« In: Gender Forum 2 (2008). www.gen derforum.org (30.06.2016).

Broeck, Sabine: Property: Gender and Slavery. In: Gender Forum. An Internent Journal for Gender Studies 14, (2006). www.genderforum.org/issues/raceing-questions-iii/ property/?print=1 (30.06.2016).

Bush, Barbara (1981): White ıladies`, coloured ‘favourites` and black ’wenches`; Some Considerations on Sex, Race and Class Factors in Social Relations in White Creole Society in the British Caribbean. In: Slavery \& Abolition: A Journal of Slave and Post-Slave Studies, Vol. 2 (3), 245-262.

Dannecker, Petra (2005): Transnational Migration and the Transformation of Gender Relations: The Case of Bangladeshi Labor Migrants. Current Sociology 53 (4): 655-674.

Dietze, Gabriele: Deutsche Exzeptionalismen - Willkomenskultur und Austerität, In: Blog Feministische Studien, 07.09.2015. http://blog.feministische-studien.de/author/ gabi-dietze/ (30.06.2016).

Dietze, Gabriele: Der okzidentalistische Geschlechterpakt. Emanzipation als uneingelöstes Versprechen. In: polar 9, Herbst 2010. www.polar-zeitschrift.de/_09.php?id=441\#441 (30.06.2016).

Dobrowolsky, Alexandra / Tastsoglou, Evangelia (2006): „Crossing boundaries and making connections". In: Dobrowolsky, Alexandra/Tastsoglou, Evangelia (Hrsg.): Women, Migration and Citizenship: Making Local, National, and Transnational Connections. Farnham, 1-35.

Ehrenreich, Barbara / Hochschild, Arlie (Hrsg.) (2004): Global Woman: Nannies, Maids, and Sex Workers in the New Economy. New York. 
Forestell, Nancy Margaret/ Moynagh, Maureen Anne (Hrsg.) (2012): General Introduction. Documenting First Wave Feminisms. In: Forestell, Nancy Margaret/Moynagh, Maureen Anne (Hrsg.): Documenting First Wave Feminisms. Volume I: Transnational Collaborations and Crosscurrents. Toronto, xix-xxv.

Franco, Jean (1999): La Malinche: From Gift to Sexual Contract (1992). In: Pratt, Mary Louise/Newman, Kathleen (Hrsg.): Critical Passions: Jean Franco: Selected Essays. Durham and London. 66-82.

Goring, Darlene (2006): The history of slave marriage in the United States. In: Journal Articles. Paper 262.

Gutiérrez Rodríguez, Encarnación (2010): Migration, Domestic Work and Affect. A Decolonial Approach on Value and the Feminization of Labor, New York.

Hanagan, Michael (2002): Gewalt und die Entstehung von Staaten. In: Wilhelm Heitmeyer/Michael Hanagan (Hrsg.): Internationales Handbuch der Gewaltforschung. Wiesbaden: $153-176$.

Isin, Engin F. / Nielsen, Greg M. (Hrsg.) (2008): Acts of Citizenship, London.

Lugones, Maria (2008): The coloniality of gender. In: Worlds and Knowledges Otherwise. Spring 2008. 1-17.

Lugones, Maria (2007): Heterosexualism and the colonial/modern gender system. In: Hypatia 22(1): 186-209.

Mamozai, Martha (1982): Herrenmenschen: Frauen im deutschen Kolonialismus. Reinbek.

Morrison, Karen Y. (2010): Slave mothers and white fathers: Defining family and status in Late Colonial Cuba. Slavery \& Abolition 31(1): 29-55.

McClintock, Anne (1995): Imperial Leather: Race, Class and Sexuality in the Colonial Context. New York/London.

Messinger, Irene (2013): There is something about marrying ... The case of human rights vs. migration regimes using the example of Austria. In: Laws 2: 376-391.

Messinger, Irene (2012): Schein oder nicht Schein. Konstruktion und Kriminalisierung von `Scheinehen in Geschichte und Gegenwart. Reihe Kritik \& Utopie. Wien.

Mies, Maria (1996): Patriarchy and Accumulation on a World Scale. London.

Mignolo, Walter D. (2000): Local Histories, Global Designs. Coloniality, Subaltern Knowledges, and Border Thinking. Princeton.

O'Connell Davidson, Julia (2001): The sex tourist, the expatriate, his ex-wife and her sother: The politics of loss, difference and desire. In: Sexualities 4(1): 5-24.

O’Connell Davidson, Julia / Sánchez Taylor Jacqueline (1999): Fantasy islands: Exploring the demand for sex tourism. In: Kempadoo, K. (Hrsg.): Sun, Sex and Gold: Tourism and Sex Work in the Caribbean. Lanham, MD, 37-54.

Parvulescu, Anca (2014): The Traffic in Women's Work: East European Migration and the Making of Europe. Chicago.

Pearce, Diana (1978): The Feminization of Poverty: Women. Work, and Welfare. In: Urban and Social Science Review, 11, 28-36.

Pelizzon, Sheila (1998): But can she spin? The decline in the social standing of women in the transition from feudalism to capitalism. PhD Dissertation, State University of New York at Binghamton.

Pruit, Deborah/LaFont, Suzanne (1995): For love and money: Romance tourism in Jamaica. In: Annals of Tourism Research 22(2): 422-440. 
Quijano, Aníbal (2000): Coloniality of power, Eurocentrism, and Latin America. In: Nepantla: Views from South 1(3): 533-574.

Roth, Julia (2013): Entangled inequalities as intersectionalities: Towards an epistemic sensibilization. In: desiguALdades.net Working Paper No. 43. www.desigualdades.net/ bilder/Working_Paper/43_WP_Roth_Online.pdf?1367229865 (30.06.2016).

Rubin, Gayle (1975): The traffic in women: Notes on the spolitical economy of sex. In: Reiter, Rayna R. (Hrsg.): Toward an Anthropology of Women. New York,157-210.

Sánchez Taylor, Jacqueline (2010): Sex tourism and inequalities. In: Cole, Stroma / Morgan, Nigel (Hrsg.): Tourism and Inequality: Problems and Prospects. Wallingford/ Cambridge, MA.

Shachar, Ayelet (2009): The Birthright Lottery: Citizenship and Global Inequality. Cambridge, MA/London.

Shohat, Ella/Stam, Robert (2012): Race in Translation: Culture Wars around the Postcolonial Atlantic. New York.

Stoler, Ann Laura / Cooper, Frederick (Hrsg.) (1997): Tensions of Empire: Colonial Cultures in a Bourgeois World. Berkeley.

Vereinte Nationen (2000): UN Milleniumsziele. www.armut.de/bekaempfung-der-armut_ internationale-strategien_un-millenniumsziele.php (30.06.2016).

Wallerstein, Immanuel (1974): The Modern World-System. Capitalist Agriculture and the Origins of the European World-Economy in the Sixteenth Century. New York.

Wallerstein, Immanuel (2003): Citizens all? Citizens some! The Making of the Citizen. Comparative Studies in Society and History, 45: 4, 650-679.

Werbner, Pnina/Yuval-Davis, Nira (1999): Introduction: Women and the new discourse on citizenship. In: Werbner, Pnina/Yuval-Davis, Nira (Hrsg.) Women, Citizenship, and Difference. New York/London. 